\title{
An Integrated Autoencoder-Based Filter for Sparse Big Data
}

\author{
Wei Peng1, Baogui Xin ${ }^{1 *}$ \\ ${ }^{1}$ College of Economics and Management, Shandong University of Science and Technology, Qingdao \\ 266590, China. \\ * Correspondence: \\ Corresponding Author \\ xin@tju.edu.cn
}

Keywords: Sparse big data, Integrated autoencoder (IAE), Data sparsity, Prediction model, Filter

\begin{abstract}
We propose a novel filter for sparse big data, called an integrated autoencoder (IAE), which utilizes auxiliary information to mitigate data sparsity. The proposed model achieves an appropriate balance between prediction accuracy, convergence speed, and complexity. We implement experiments on a GPS trajectory dataset, and the results demonstrate that the IAE is more accurate and robust than some state-of-the-art methods.
\end{abstract}

\section{Introduction}

\subsection{Motivation}

Prediction is essential in many scientific fields, such as economics [1-7], engineering [8-12], and medicine [13-16]. A valid prediction can facilitate sound decisions. But high sparsity of original data can substantially decrease prediction performance. Existing prediction algorithms [17-21] are not good at processing such data. We may leverage auxiliary information to mitigate the sparsity of data, but these algorithms have difficulty representing complicated features when auxiliary data are unstructured and heterogeneous. The deep learning-based scheme can extract complex nonlinear representations from these data, and it usually shows better prediction performance.

Deep learning has led to remarkable achievements in fields such as computer vision [22-25], speech recognition [26-30], and natural language processing [31, 32], which have attracted much attention in theory and practice. Many deep learning frameworks have been developed, such as the convolutional neural network (CNN) [24, 33-35], recurrent neural network (RNN) [36-39], long short-term memory (LSTM) [1, 32, 40, 41], deep belief network (DBN) [6, 8, 42, 43], AE [9, 44-47] and so on. In some circumstances, deep learning frameworks deal inefficiently with unstructured and heterogeneous data, because data preprocessing increases the complexity of the structure.

The AE has low complexity and no data preprocessing requirement. AEs are widely applied in prediction [2, 9, 11, 48], classification [49-52], and latent representation learning [44, 45, 53, 54]. Various requirements give rise to many variations of AEs. Vincent et al. [44, 45] proposed a denoising AE (DAE), stack AEs (SAE), and stack denoising AEs (SDAE), which focus on extracting more abstract representations for a standalone supervised deep learning architecture. Görgel and Simsek [12] 
developed deep stacked denoising sparse autoencoders (DSDSA) for face recognition and the sparsity property of DSDSA substantially decreased the possibility of overfitting. Lv et al. [55] applied an SAE to tourism demand prediction using search query data, which has a better performance than some other state-of-the-art deep learning-based models. The SAE and SDAE are stacked by the AE and DAE and obtain their optimal values through several optimization processes, which lead to low efficiency. In brief, the above variations of the AE obtain higher accuracy by increasing complexity and decreasing efficiency.

\subsection{Contributions}

Autoencoder (AE), stacked AE (SAE), denoising AE (DAE) and stacked DAE (SDAE) are efficient prediction approaches with low complexity that has been widely applied in some scientific fields. However, to obtain a well-trained SAE or SDAE stacked by $k$ AEs or DAEs, we must optimize $k+1$ objective functions, which require a significant amount of computing resources. By integrating autoencoders, we develop a novel filter only by optimizing a single objective function. In addition, the integrated autoencoder (IAE) utilize auxiliary information to mitigate data sparsity to achieve an appropriate balance among prediction accuracy, convergence speed, and complexity. Experiments demonstrate that the proposed IAE performs superior than above-mentioned model in accuracy and efficiency.

\subsection{Framework}

The remainder of this paper is organized as follows. Section 2 reviews some related prediction methods. Section 3 describes the IAE in detail. Section 4 discusses experiments and results. Section 5 summarizes conclusions.

\section{Related Work}

\subsection{Non-AE prediction methods}

Machine learning-based prediction methods can effectively deal with nonlinear prediction problems. They consist mainly of singular value decomposition (SVD) [56, 57], matrix factorization (MF) [18, 58], support vector machine (SVM) [59, 60], and Bayesian models [61, 62]. Machine learning-based

prediction can enhance prediction performance to some extent. However, it is hard to obtain a substantial breakthrough.

Deep learning-based methods are typically composed of numerous neurons and parameters, which can extract more complicated and nonlinear features from the input data. For example, Krizhevsky et al. [24] proposed a deep CNN with 60 million parameters and 650,000 neurons to classify 1000 image categories. Since the dataset they employed contained 1.2 million images, the deep CNN architecture could prevent overfitting to some extent and performed better than previous deep learning-based methods. However, it was too complicated to train quickly. Ting et al. [33] proposed a CNN-based architecture (CNNI-BCC) for breast cancer classification. Compared with previous work, CNNI-BCC is indeed more accurate. However, it contains 30 hidden layers and each layer contains lots of parameters, which leads to low efficiency. Chan et al. [63] proposed a simpler unsupervised convolutional neural network (PCANet) with fewer parameters, which could accelerate training, but whose performance was significantly degraded when the images were not well prepared. Song et al. [64] developed a content-based automatic tagging algorithm with five-layer RNNs which performed a higher training efficiency than above-mentioned algorithms with numerous layers and parameters. But the model need a preprocessing phase, which also takes too much time. LSTM, as an extension of 
RNN, is a more sophisticated but effective structure with a gating mechanism. Baek and Kim [1] proposed a data-augmentation approach (ModAugNet) consisting of an overfitting prevention LSTM and a prediction LSTM, which can avoid overfitting by overcoming the deficiency of limited data availability. Mohamed et al. [65] replaced Gaussian mixture models by DBNs for acoustic modelling and achieved a better performance. But the architecture contained lots of hidden neurons and parameters in every layer, which could impact its convergence speed.

\subsection{AE and its Variations}

Though the above methods can significantly improve prediction performance, most are complicated, which may cause low efficiency. The AE is a simple but effective deep learning approach that can learn complex representations with high efficiency.

\subsection{1 $A E$}

An $\mathrm{AE}$ is an unsupervised representation learning framework with three parts: the input layer, hidden layer, and output layer, which are defined as

$$
\begin{gathered}
H=f_{\theta}(x)=f(W \times x+b) \\
O=g_{\theta^{\prime}}(H)=g\left(W^{\prime} \times H+b^{\prime}\right)
\end{gathered}
$$

where $x$ is the original input; $H$ is the hidden representation; $O$ is the output; $f(\cdot)$ and $g(\cdot)$ are encoder and decoder activation functions, respectively; $W$ and $W^{\prime}$ are weights; $b$ and $b^{\prime}$ are biases; $\theta=\{W, \mathrm{~b}\} ;$ and $\theta^{\prime}=\left\{W^{\prime}, b^{\prime}\right\}$. Figure 1 describes the architecture of an AE.

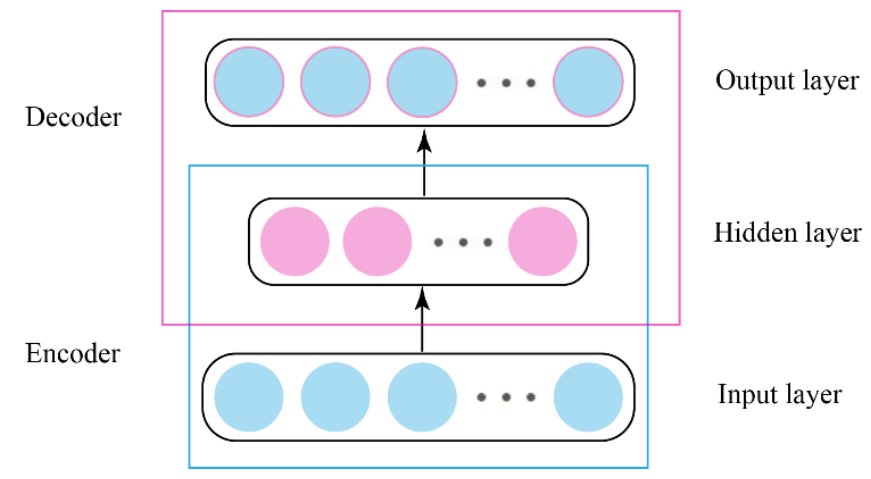

Figure 1: Architecture of an AE.

To obtain optimal parameters by minimizing the error between input and output layers, we write the following loss function of the AE:

$$
e_{A E}=\min \sum\left\|x-g\left(W^{\prime} \times f(W \times x+b)+b^{\prime}\right)\right\|_{\mathcal{O}}^{2}
$$

where \|\|$_{\mathcal{O}}^{2}$ denotes that we consider only the observed data.

The AE can learn latent representations by simply copying input, but the learned representations are usually too specific to generalize well. In some scenarios, one must enhance the generalization ability 
by stacking several AEs, which can learn more representative features than a single AE. Also, for different demands, one must propose some variations of the AE.

Thus some researchers have combined the AE with other deep learning models to both improve efficiency and accuracy. AE-based methods perform better with sparse data, such as rating data in recommender systems. Sedhain et al. [66] proposed an AE-based collaborative model (AutoRec), which has advantages over existing neural network approaches in representing and computing. Lv et al. [9] applied an SAE to traffic prediction, which is indispensable to better city planning.

\subsubsection{Stacked AE (SAE)}

An SAE stacks several typical AEs to attain stronger generalization ability. Its training process includes pre-training and fine-tuning, as shown in Figure 2.

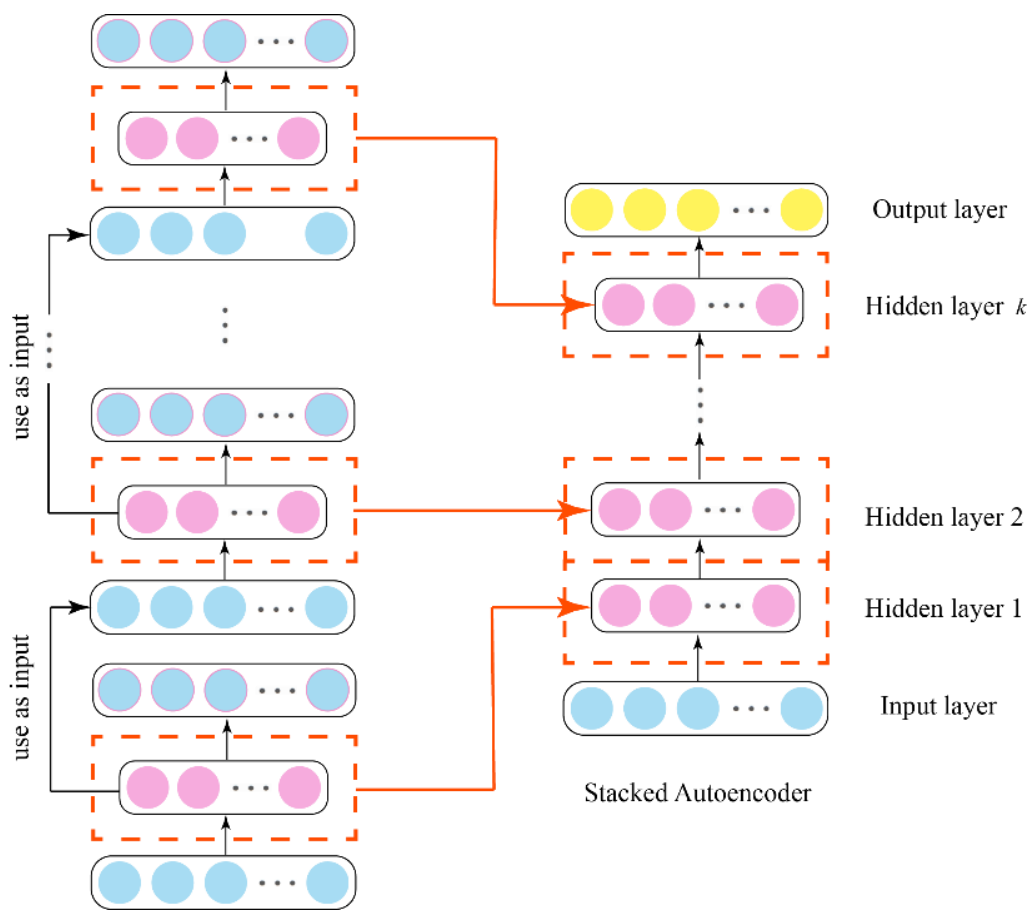

Figure 2: Training process of SAE.

According to Figure 2, assuming there is an SAE stacked by $k$ AEs, the output of each AE in the pretraining stage is discarded, and each hidden layer is used as the input of the next $\mathrm{AE}$ until the $k^{\text {th }} \mathrm{AE}$ is well trained. After pre-training each $\mathrm{AE}$, the original input is transformed to the top $\mathrm{AE}$, layer by layer. The hidden layer of each AE can be calculated by

$$
H_{i}= \begin{cases}g^{(1)}\left(W_{1}^{\prime} \times f^{(1)}\left(W_{1} \times x+b_{1}\right)+b_{1}^{\prime}\right), & i=1 \\ g^{(i)}\left(W_{i}^{\prime} \times f^{(i)}\left(W_{i} \times H_{i-1}+b_{i}\right)+b_{i}^{\prime}\right), & i=2, \cdots, k\end{cases}
$$

where $H_{i}$ represents the hidden layer of the $i^{\text {th }} \mathrm{AE} ; f^{(i)}$ and $g^{(i)}$ respectively represent the encoder and decoder activation functions of the $i^{\text {th }} \mathrm{AE} ; W_{i}$ and $W_{i}^{\prime}$ respectively represent the encoder and decoder weight of the $i^{\text {th }} \mathrm{AE}$; and $b_{i}$ and $b_{i}^{\prime}$ respectively represent the encoder and decoder bias of the $i^{\text {th }} \mathrm{AE}$. 
The top hidden layer of the SAE can be taken as input to a standalone supervised learning approach. For example, to finish classification tasks, one often adds a softmax classification layer connected to the top hidden layer. In the fine-tuning stage, to obtain the fine-tuned parameters by minimizing the reconstruction error, the loss function is defined by

$$
e_{S A E}=\min \left\{\| x-\left.\hat{x}\right|_{\mathcal{O}} ^{2} \mid\left\{\min \left(e_{A E}^{(k)}\right) \mid\left\{\min \left(e_{A E}^{(k-1)}\right) \mid\left\{\cdots \mid \min \left(e_{A E}^{(1)}\right)\right\}\right\}\right\}\right\}
$$

where $e_{A E}^{(k)}$ denotes the reconstruction error of the $k^{\text {th }} \mathrm{AE}$.

Though the SAE has better generalization ability, its performance can be affected when the original input is mixed with noise. In some circumstances, an AE needs a denoised input.

\subsubsection{Denoising $A E(D A E)$}

A DAE reconstructs a repaired (or "clean") input from a partially destroyed (or "corrupted") one. It is achieved by first partially destroying the raw input $x$ to obtain a corrupted version $\tilde{x}$ via a stochastic mapping $\tilde{x} \sim q_{D}(\tilde{x} \mid x)$. And then $\tilde{x}$ is mapped by an AE, which includes the encoding process $h=f_{\theta}(\tilde{x})=f(W \tilde{x}+b)$ and the decoding process $\hat{x}=g_{\theta^{\prime}}(y)=g\left(W^{\prime} h+b^{\prime}\right)$. Figure 3 illustrates the mechanism of a DAE.

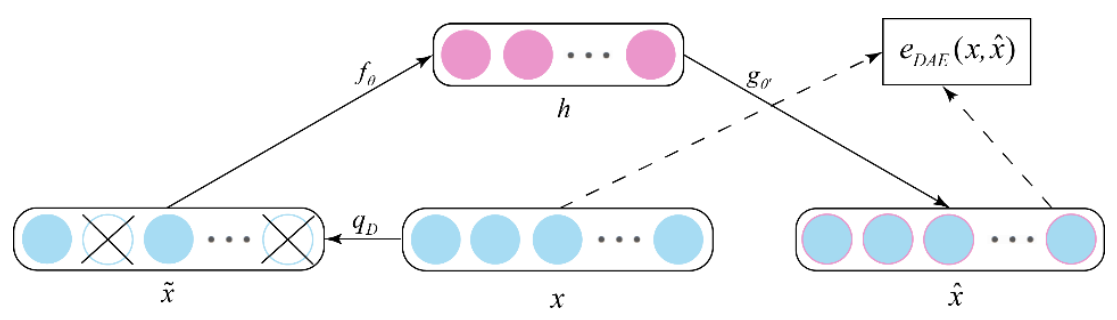

Figure 3: Mechanism of a DAE.

Similarly, parameters are optimized by the following loss function, which minimizes the reconstruction error between the raw input $x$ and output $z$ :

$$
e_{D A E}=\min \sum\left\|x-g\left(W^{\prime} \times f(W \times \tilde{x}+b)+b^{\prime}\right)\right\|_{\mathcal{O}}^{2}
$$

Indeed, it is natural to take into account stacking DAEs to obtain a more powerful AE-based model by exploiting their respective advantages.

\subsubsection{Stacking DAE (SDAE)}

Stacking DAEs or typical AEs to build a deep network is the same as stacking RBMs in deep belief networks. The training process of an SDAE also includes pre-training and fine-tuning. The structure of an SDAE is shown in Figure 4. 


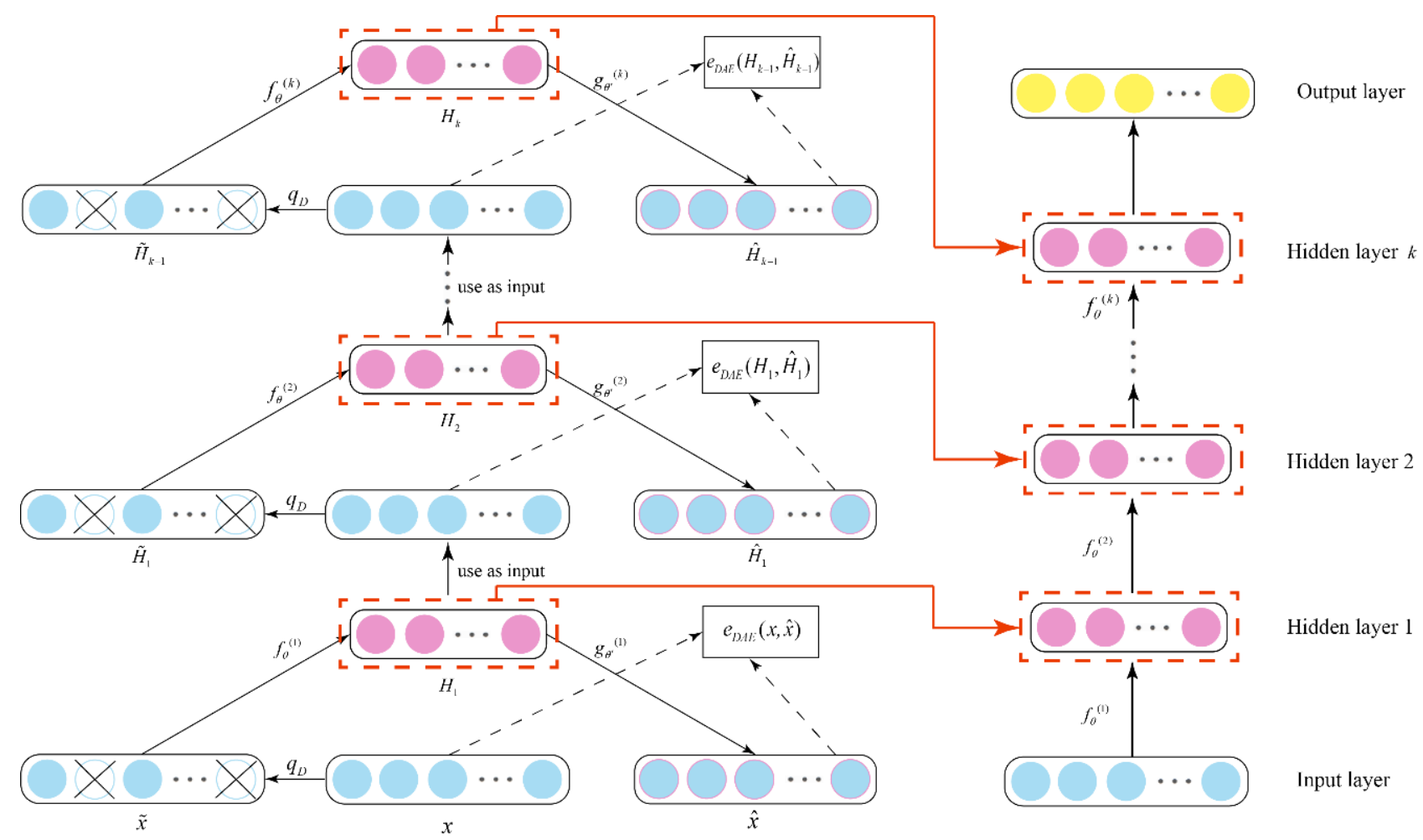

Figure 4: Training process of SDAE.

In the pre-training stage, the output of each DAE is dropped, and each hidden layer is used as the input of the next DAE until the $k^{\text {th }}$ DAE is well trained. Similarly, to finish classification tasks, one often adds a softmax classification layer as an output layer connected to the top hidden layer. In the finetuning stage, to obtain the fine-tuned parameters by minimizing the reconstruction error, the loss function is defined by

$$
e_{S D A E}=\min \left\{\|x-\hat{x}\|_{\mathcal{O}}^{2} \mid\left\{\min \left(e_{D A E}^{(k)}\right)\left\{\min \left(e_{D A E}^{(k-1)}\right) \mid\left\{\cdots \mid \min \left(e_{D A E}^{(1)}\right)\right\}\right\}\right\}\right\}
$$

where $e_{D A E}^{(k)}$ denotes the reconstruction error of the $k^{\text {th }}$ DAE.

There are some previous works based on above-mentioned SAE and SDAE. Tong et al. [11] developed a novel architecture which makes use of SDAEs and ensemble learning (SDAEsTSE) for software defect prediction. And the SDAEsTSE achieves a good balance between accuracy and efficiency. Bai et al. [67] proposed a DL-SSAE model which takes into account seasonality to predict PM 2.5 concentration. And the experimental results demonstrate that the DL-SSAE outperforms some stateof-the-art models whether or not to consider seasonality. To sum up, the AE is an efficient prediction approach with low complexity that has been widely applied in some scientific fields.

\subsubsection{Summary}

There is no doubt that SAE and SDAE can improve prediction accuracy. However, to obtain a welltrained SAE or SDAE stacked by $k$ AEs or DAEs, we must optimize $k+1$ objective functions, which will consume a lot of computing resources. Therefore, it is urgent to develop a new variation of AE to 
overcome the shortcoming caused by multiply objective functions. In the next Section, we will develop a novel filter only by optimizing a single objective function.

\section{Integrated autoencoder (IAE)}

In this section, we introduce a novel filter with a single objective function for sparse big data, called IAE, which utilizes auxiliary information to mitigate data sparsity. The IAE model joins two AEs to enhance both accuracy and efficiency. The structure of the IAE is depicted in Figure 5.

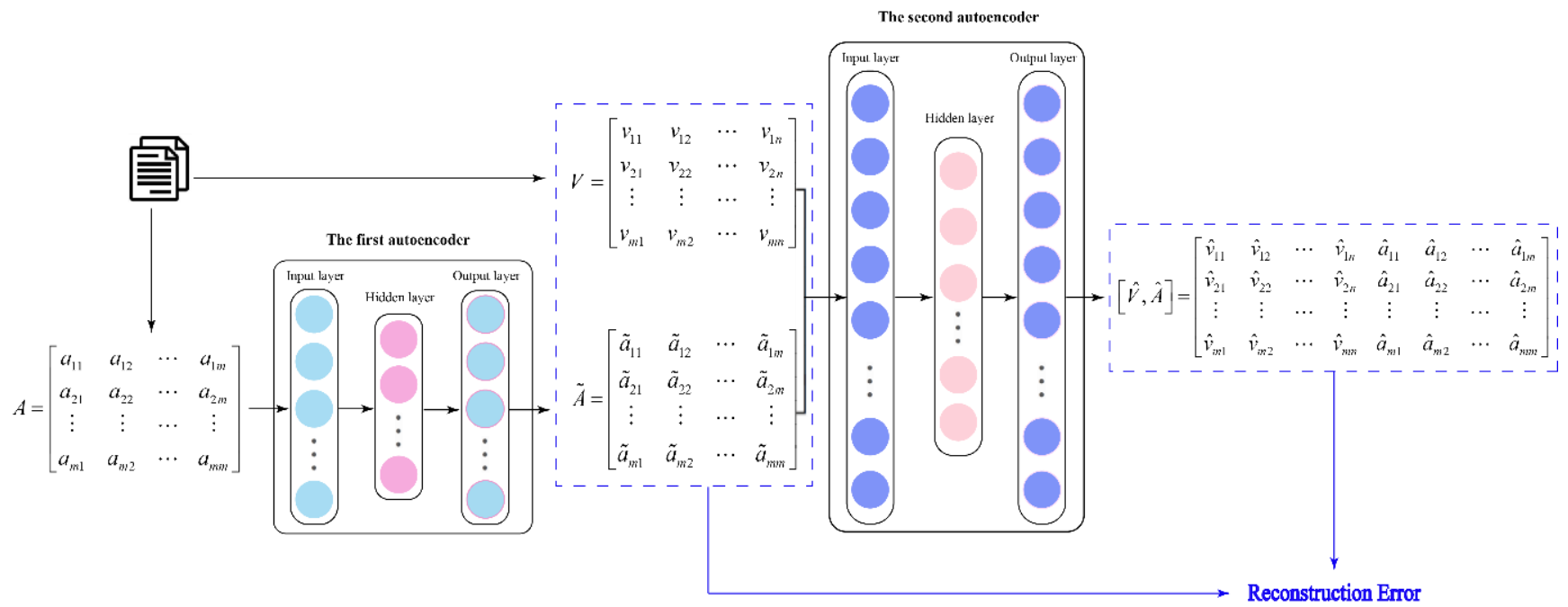

Figure 5: Structure of the proposed IAE model.

Assume that there is a matrix $V \in \mathbb{R}^{m \times n}$ that must be predicted, and an auxiliary information matrix $A \in \mathbb{R}^{m \times m}$ that can be employed to enhance the prediction accuracy of $V$.

At the first stage, we feed $A$ as the input of the first AE, which will perform representation learning from $A$ and output a reduction version $\tilde{A}$.

$$
\tilde{A}=g^{(1)}\left(W_{1}^{\prime} \times f^{(1)}\left(W_{1} \times A+b_{1}\right)+b_{1}^{\prime}\right)
$$

At the second stage, we concatenate $V$ and $\tilde{A}$ horizontally to generate a new matrix $[V, \tilde{A}]$ which is fed as the input of the second AE. Similarly, the second AE performs representation learning from $[V, \tilde{A}]$ and derives a predicted matrix $[\hat{V}, \hat{A}]$. We take the first $n$ columns of $[\hat{V}, \hat{A}]$ as the predicted matrix $\hat{V}$.

Also, we leverage the back-propagation algorithm to minimize the reconstruction error between $V$ and $\hat{V}$ at the second stage. To prevent overfitting, we add the regularization term to the following loss function:

$$
e_{J A E}=\min \sum\left\|[V, \tilde{A}]-g^{(2)}\left(W_{2}^{\prime} \times f^{(2)}\left(W_{2} \times[V, \tilde{A}]+b_{2}\right)+b_{2}^{\prime}\right)\right\|_{\mathcal{O}}^{2}+\frac{\lambda}{2}\left(\left\|W_{2}\right\|_{F}^{2}+\left\|W_{2}^{\prime}\right\|_{F}^{2}\right)
$$

where $\lambda$ is a hyper parameter. 
In the IAE, the second AE implements the error minimization between $[V, \tilde{A}]$ and $[\hat{V}, \hat{A}]$, while the first $\mathrm{AE}$ does not perform the error minimization between $A$ and $\tilde{A}$. What is more, there are only two hidden layers in an IAE, and it needs no pre-processing. So, the IAE must consume less running time than the SAE, DAE, or SDAE.

\section{$4 \quad$ Experiments}

\subsection{Experimental setup}

\subsubsection{Datasets}

We conducted experiments on the GPS trajectory dataset, which contains real-time traffic conditions and POI features of Beijing. Our purpose is to take POI (point of interest) features as auxiliary information to predict the traffic conditions $\bar{v}_{r, t}$ and $s d v_{r, t}$, which respectively denote the average travel speed and standard deviation of the travel speed of all of the vehicles traversing road segment $r$ in the time slot $t$.

The GPS trajectory dataset also has been employed [68]; this is a context-aware matrix factorization method to predict gas consumption and pollution emissions via the prediction of $\bar{v}$ and $s d v$. Moreover, it can be widely used for urban computing scenarios [69].

To verify the performance of the IAE, we randomly divided the experimental dataset into two parts, a training dataset $D_{\text {train }}$ and testing dataset $D_{\text {test }}$, accounting for $\varphi \%$ and $1-\varphi \%$, respectively.

\subsubsection{Evaluation Index}

Root mean square error (RMSE) is a widely used evaluation index for recommender systems or continuous type prediction. By calculating the RMSE between observed values and predicted values on $D_{\text {test }}$, we can test the accuracy of the IAE. A smaller RMSE value represents better performance. It is defined as

$$
R M S E=\sqrt{\frac{\sum_{(r, t) \in D_{\text {test }}}\left(\bar{v}_{r, t}-\hat{\bar{v}}_{r, t}\right)^{2}}{\left|D_{\text {test }}\right|}}
$$

where $\hat{\bar{v}}_{r, t}$ represents the predicted value of $\bar{v}_{r, t}$, and $|\cdot|$ represents the number of elements in a set.

The efficiency is measured by the average time $A_{t}$ consumed in each epoch of the program:

$$
A_{t}=\frac{T_{t}}{n_{\text {epochs }}}
$$

where $T_{t}$ denotes the total time consumed in the program, and $n_{\text {epochs }}$ denotes the number of epochs in the program.

\subsubsection{Experimental Implementation and Parameter Settings}

We performed all experiments in Python on a PC with a $2.00 \mathrm{GHz}$ CPU, 16 GB memory, and an NVIDIA GTX 1080Ti-11G GPU. 
We adopted the Adam optimizer to minimize the reconstruction error and set some important parameters, as shown in Table 1.

Table 1: Parameter settings.

\begin{tabular}{llll}
\hline & Parameters & $\bar{v}$ & $s d v$ \\
\hline \multirow{3}{*}{ Common parameters } & Hyper-parameter & $\lambda=0.01$ & $\lambda=0.01$ \\
& Training ratio & $\varphi=80$ & $\varphi=80$ \\
& Training epochs & $n=100$ & $n=100$ \\
AE & Number of neurons in hidden layer & 1,000 & 1,000 \\
& Number of neurons in first hidden layer & 1,000 & 1,000 \\
DAE & Number of neurons in second hidden layer & 3,000 & 3,000 \\
SDAE & Number of neurons in hidden layer & 1,000 & 1,000 \\
& Number of neurons in first hidden layer & 1,000 & 1,000 \\
IAE & Number of neurons in second hidden layer & 3,000 & 3,000 \\
& Number of neurons in first hidden layer & 1,000 & 1,000 \\
& Number of neurons in second hidden layer & 3,000 & 3,000 \\
\hline
\end{tabular}

\subsection{Experimental Results}

In this section, we set the training epochs of different AE-based algorithms to 100, and obtained the corresponding results, as shown in Table 2.

Table 2: Performance Comparison of Algorithms.

\begin{tabular}{llll}
\hline Algorithm & RMSE and $A_{t}$ & $\bar{v}$ & $s d v$ \\
\hline AE & RMSE & 3.968 & 1.374 \\
& $A_{t}$ & $4.18 \mathrm{~s}$ & $3.92 \mathrm{~s}$ \\
SAE & RMSE & 4.294 & 1.511 \\
& $A_{t}$ & $33.52 \mathrm{~s}$ & $35.75 \mathrm{~s}$ \\
DAE & RMSE & 4.054 & 1.444 \\
& $A_{t}$ & $4.14 \mathrm{~s}$ & $4.77 \mathrm{~s}$ \\
SDAE & RMSE & 4.299 & 1.508 \\
& $A_{t}$ & $29.95 \mathrm{~s}$ & $34.58 \mathrm{~s}$ \\
IAE & RMSE & 2.079 & 0.455 \\
& $A_{t}$ & $23.83 \mathrm{~s}$ & $21.44 \mathrm{~s}$ \\
\hline
\end{tabular}

Table 2 shows that the accuracy of the IAE exceeds that of other AE-based models. The efficiency of the IAE is higher than that of the SAE and SDAE. It is inevitable that the speed of the AE and DAE is higher than that of the IAE, because the AE and DAE consist of a single hidden layer while the SAE, SDAE, and IAE consist of at least two hidden layers (in this paper, we consider only the SAE, SDAE, and IAE with two hidden layers). Moreover, the SAE, DAE, and SDAE focus on extracting more 
abstract representations for a standalone supervised deep learning architecture. Therefore, it is justifiable that the RMSEs of the SAE, DAE, and SDAE are all higher than that of the AE.

Figures 6 and 7 show the trend that the accuracy of different algorithms varies with the increase of training epochs. From Figure 6, we note that the RMSEs of the IAE continue decreasing between about epoch 28 and epoch 76, while those of the AE, DAE, SAE, and SDAE decrease at the first several epochs but later fluctuate in a broad range. From Figure 7, we find that the RMSEs of the IAE continue to decrease with increased training epochs, while those of the AE, DAE, SAE, and SDAE continue fluctuating and do not show a significant downward trend. It is clear that the IAE is more accurate and robust than the AE, DAE, SAE, and SDAE.

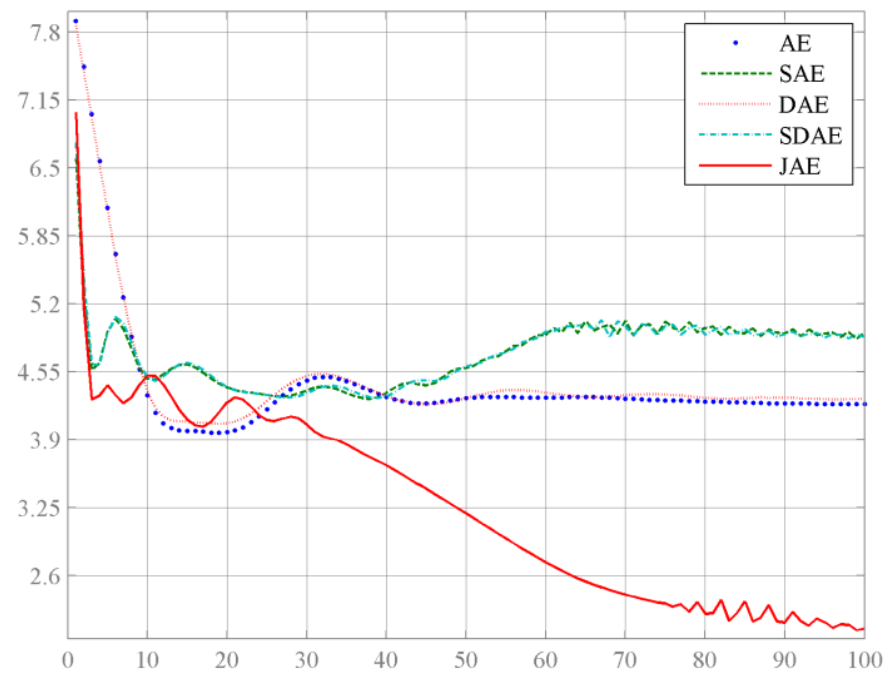

Figure 6: RMSE of $\bar{v}$.

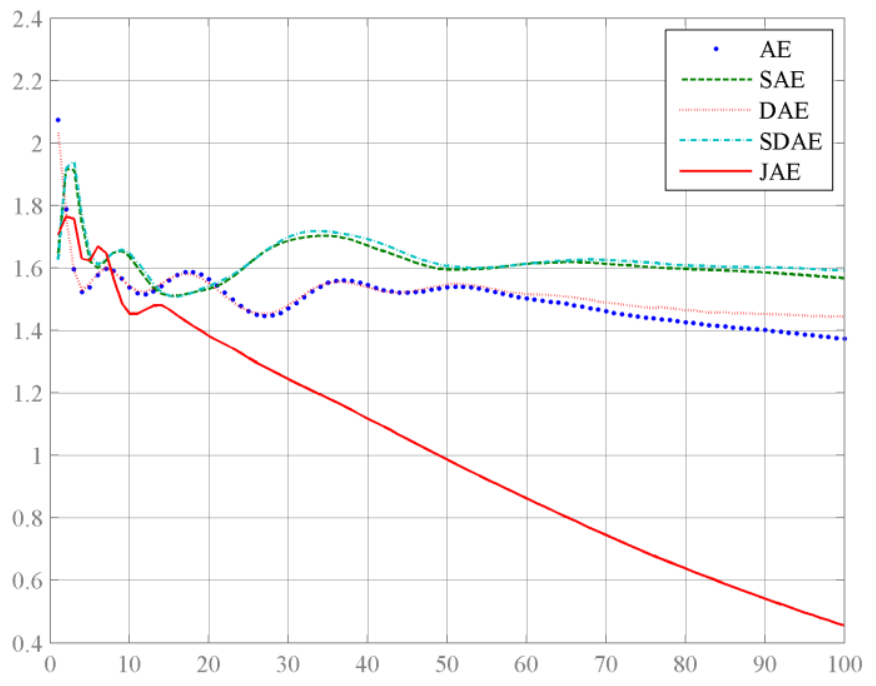

Figure 7: RMSE of $s d v$. 


\section{Conclusions}

In this paper, we built a novel filter only with a single objective function, named IAE, which utilizes auxiliary information to mitigate data sparsity. The IAE achieves an appropriate balance among prediction accuracy, convergence speed, and complexity. We regard the IAE as a deep learning structure that utilizes auxiliary information for prediction. Traffic prediction, as mentioned above, is an example that demonstrates the IAE's validity. The IAE can be used to efficiently predict big data with auxiliary information in almost all fields [70, 71].

\section{Conflict of Interest}

The authors declare that the research was conducted in the absence of any commercial or financial relationships that could be construed as a potential conflict of interest.

\section{Author Contributions}

BX: conceptualization. BX and WP: methodology. BX and WP: software. BX and WP: validation. BX: formal analysis. WP: writing-original draft preparation. BX and WP: writing-review and editing. WP: visualization. BX: supervision, project administration, and funding acquisition.

\section{Funding}

The work was supported by the National Social Science Foundation of China [No. 16FJY008]; the National Natural Science Foundation of China [No. 11801060]; and the Natural Science Foundation of Shandong Province [No. ZR2016FM26].

\section{Acknowledgments}

The authors would like to express sincere gratitude to the referees for their valuable suggestions and comments.

\section{Data Availability}

The GPS trajectory dataset used to support the findings of this study are available from the corresponding author upon request, or to directly download from the following website:

https://onedrive.live.com/?authkey=\%21ADgmvTgfqs4hn4Q\&id=CF159105855090C5\%211438\&ci $\mathrm{d}=\mathrm{CF} 159105855090 \mathrm{C} 5$.

\section{Reference}

1. Baek, Y. and H.Y. Kim, ModAugNet: A new forecasting framework for stock market index value with an overfitting prevention LSTM module and a prediction LSTM module. Expert Systems with Applications, 2018. 113: p. 457-480.

2. Wang, K., et al., SDDRS: Stacked Discriminative Denoising Auto-Encoder based Recommender System. Cognitive Systems Research, 2019. 55: p. 164-174.

3. Tsantekidis, A., et al. Forecasting stock prices from the limit order book using convolutional neural networks. in Business Informatics (CBI), 2017 IEEE 19th Conference on. 2017. IEEE. 
4. $\quad$ Fischer, T. and C. Krauss, Deep learning with long short-term memory networks for financial market predictions. European Journal of Operational Research, 2018. 270(2): p. 654-669.

5. Chai, J., et al., A Decomposition-Integration Model with Dynamic Fuzzy Reconstruction for Crude Oil Price Prediction and The Implications for Sustainable Development. Journal of Cleaner Production, 2019.

6. Shen, F., J. Chao, and J. Zhao, Forecasting exchange rate using deep belief networks and conjugate gradient method. Neurocomputing, 2015. 167: p. 243-253.

7. McGurk, Z., US Real Estate Inflation Prediction: Exchange Rates and Net Foreign Assets. The Quarterly Review of Economics and Finance, 2019.

8. Wang, K., et al., Deep belief network based k-means cluster approach for short-term wind power forecasting. Energy, 2018. 165: p. 840-852.

9. Lv, Y., et al., Traffic Flow Prediction With Big Data: A Deep Learning Approach. IEEE Transactions on Intelligent Transportation Systems, 2015. 16(2): p. 865-873.

10. $\mathrm{Xu}, \mathrm{F}$., W.t.P. Tse, and Y.L. Tse, Roller bearing fault diagnosis using stacked denoising autoencoder in deep learning and Gath-Geva clustering algorithm without principal component analysis and data label. Applied Soft Computing, 2018. 73: p. 898-913.

11. Tong, H., B. Liu, and S. Wang, Software defect prediction using stacked denoising autoencoders and two-stage ensemble learning. Information and Software Technology, 2018. 96: p. 94-111.

12. Görgel, P. and A. Simsek, Face recognition via Deep Stacked Denoising Sparse Autoencoders (DSDSA). Applied Mathematics and Computation, 2019. 355: p. 325-342.

13. Alipanahi, B., et al., Predicting the sequence specificities of DNA-and RNA-binding proteins by deep learning. Nature biotechnology, 2015. 33(8): p. 831.

14. Abraham, B. and M.S. Nair, Computer-aided diagnosis of clinically significant prostate cancer from MRI images using sparse autoencoder and random forest classifier. Biocybernetics and Biomedical Engineering, 2018. 38(3): p. 733-744.

15. Chen, M., et al., Disease prediction by machine learning over big data from healthcare communities. IEEE Access, 2017. 5: p. 8869-8879.

16. Deng, Y., et al., Towards automatic encoding of medical procedures using convolutional neural networks and autoencoders. Artificial Intelligence in Medicine, 2019. 93: p. 29-42.

17. Urso, A., et al., Data Mining: Prediction Methods, in Encyclopedia of Bioinformatics and Computational Biology, S. Ranganathan, et al., Editors. 2019, Academic Press: Oxford. p. 413-430.

18. Koren, Y., R. Bell, and C. Volinsky, Matrix factorization techniques for recommender systems. Computer, 2009(8): p. 30-37.

19. Hrasko, R., A.G.C. Pacheco, and R.A. Krohling, Time Series Prediction Using Restricted Boltzmann Machines and Backpropagation. Procedia Computer Science, 2015. 55: p. 990999.

20. Cong, Y., J. Wang, and X. Li, Traffic Flow Forecasting by a Least Squares Support Vector Machine with a Fruit Fly Optimization Algorithm. Procedia Engineering, 2016. 137: p. 59-68. 
21. Tang, J., et al., Traffic flow prediction based on combination of support vector machine and data denoising schemes. Physica A: Statistical Mechanics and its Applications, 2019.

22. Mathews, S.M., C. Kambhamettu, and K.E. Barner, A novel application of deep learning for single-lead ECG classification. Computers in Biology and Medicine, 2018. 99: p. 53-62.

23. Elmahmudi, A. and H. Ugail, Deep face recognition using imperfect facial data. Future Generation Computer Systems, 2019. 99: p. 213-225.

24. Krizhevsky, A., I. Sutskever, and G.E. Hinton. ImageNet Classification with Deep Convolutional Neural Networks. in International Conference on Neural Information Processing Systems. 2012.

25. Beyaz, A., et al., Olive fly sting detection based on computer vision. Postharvest Biology and Technology, 2019. 150: p. 129-136.

26. Hinton, G., et al., Deep neural networks for acoustic modeling in speech recognition: The shared views of four research groups. IEEE Signal processing magazine, 2012. 29(6): p. 8297.

27. Fayek, H.M., M. Lech, and L. Cavedon, Evaluating deep learning architectures for Speech Emotion Recognition. Neural Networks, 2017. 92: p. 60-68.

28. Grozdić, Đ.T., S.T. Jovičić, and M. Subotić, Whispered speech recognition using deep denoising autoencoder. Engineering Applications of Artificial Intelligence, 2017. 59: p. 1522.

29. Tu, Y.-H., et al., An iterative mask estimation approach to deep learning based multi-channel speech recognition. Speech Communication, 2019. 106: p. 31-43.

30. Zhao, J., X. Mao, and L. Chen, Speech emotion recognition using deep $1 D$ \& $2 D$ CNN LSTM networks. Biomedical Signal Processing and Control, 2019. 47: p. 312-323.

31. Araque, O., et al., Enhancing deep learning sentiment analysis with ensemble techniques in social applications. Expert Systems with Applications, 2017. 77: p. 236-246.

32. Wang, J., B. Peng, and X. Zhang, Using a stacked residual LSTM model for sentiment intensity prediction. Neurocomputing, 2018. 322: p. 93-101.

33. Ting, F.F., Y.J. Tan, and K.S. Sim, Convolutional neural network improvement for breast cancer classification. Expert Systems with Applications, 2019. 120: p. 103-115.

34. Abid, F., et al., Sentiment analysis through recurrent variants latterly on convolutional neural network of Twitter. Future Generation Computer Systems, 2019. 95: p. 292-308.

35. Steinbrener, J., K. Posch, and R. Leitner, Hyperspectral fruit and vegetable classification using convolutional neural networks. Computers and Electronics in Agriculture, 2019. 162: p. 364-372.

36. Liu, Z. and C.J. Sullivan, Prediction of weather induced background radiation fluctuation with recurrent neural networks. Radiation Physics and Chemistry, 2019. 155: p. 275-280.

37. Pan, Y., et al., Predicting bike sharing demand using recurrent neural networks. Procedia Computer Science, 2019. 147: p. 562-566.

38. Zagrebina, S.A., V.G. Mokhov, and V.I. Tsimbol, Electrical Energy Consumption Prediction is based on the Recurrent Neural Network. Procedia Computer Science, 2019. 150: p. 340346. 
39. Naghibi, Z., S.A. Sadrossadat, and S. Safari, Time-domain modeling of nonlinear circuits using deep recurrent neural network technique. AEU - International Journal of Electronics and Communications, 2019. 100: p. 66-74.

40. Lei, J., C. Liu, and D. Jiang, Fault diagnosis of wind turbine based on Long Short-term memory networks. Renewable Energy, 2019. 133: p. 422-432.

41. Cen, Z. and J. Wang, Crude oil price prediction model with long short term memory deep learning based on prior knowledge data transfer. Energy, 2019. 169: p. 160-171.

42. Hassan, M.M., et al., Human emotion recognition using deep belief network architecture. Information Fusion, 2019. 51: p. 10-18.

43. Chen, G., et al., Predict effective drug combination by deep belief network and ontology fingerprints. Journal of Biomedical Informatics, 2018. 85: p. 149-154.

44. Vincent, P., et al. Extracting and composing robust features with denoising autoencoders. in International Conference on Machine Learning. 2008.

45. Vincent, P., et al., Stacked Denoising Autoencoders: Learning Useful Representations in a Deep Network with a Local Denoising Criterion. Journal of Machine Learning Research, 2010. 11(12): p. 3371-3408.

46. Jia, F., et al., A neural network constructed by deep learning technique and its application to intelligent fault diagnosis of machines. Neurocomputing, 2018. 272: p. 619-628.

47. Bao, W., J. Yue, and Y. Rao, A deep learning framework for financial time series using stacked autoencoders and long-short term memory. PloS one, 2017. 12(7): p. e0180944.

48. Mahdi, M. and V.M.I. Genc, Post-fault prediction of transient instabilities using stacked sparse autoencoder. Electric Power Systems Research, 2018. 164: p. 243-252.

49. Ng, W.W.Y., et al., Dual autoencoders features for imbalance classification problem. Pattern Recognition, 2016. 60: p. 875-889.

50. Kannadasan, K., D.R. Edla, and V. Kuppili, Type 2 diabetes data classification using stacked autoencoders in deep neural networks. Clinical Epidemiology and Global Health, 2018.

51. Fu, X., et al., Semi-supervised Aspect-level Sentiment Classification Model based on Variational Autoencoder. Knowledge-Based Systems, 2019. 171: p. 81-92.

52. Zhang, L., et al., PolSAR image classification based on multi-scale stacked sparse autoencoder. Neurocomputing, 2019.

53. Ohno, H., Linear guided autoencoder: Representation learning with linearity. Applied Soft Computing, 2017. 55: p. 566-575.

54. Zhuang, F., et al., Representation learning via Dual-Autoencoder for recommendation. Neural Networks, 2017. 90: p. 83-89.

55. Lv, S.-X., L. Peng, and L. Wang, Stacked autoencoder with echo-state regression for tourism demand forecasting using search query data. Applied Soft Computing, 2018. 73: p. 119-133.

56. Tănăsescu, A. and P.G. Popescu, A fast singular value decomposition algorithm of general ktridiagonal matrices. Journal of Computational Science, 2019. 31: p. 1-5.

57. Busu, C. and M. Busu, Modelling the predictive power of the singular value decomposition based-entropy. Empirical evidence from New York Stock Exchange. Physica A: Statistical Mechanics and its Applications, 2019. 
58. Belohlavek, R., J. Outrata, and M. Trnecka, Toward quality assessment of Boolean matrix factorizations. Information Sciences, 2018. 459: p. 71-85.

59. $\mathrm{Xu}, \mathrm{Z}$., et al., A regression-type support vector machine for $k$-class problem. Neurocomputing, 2019. 340: p. 1-7.

60. Karimi, F., et al., An enhanced support vector machine model for urban expansion prediction. Computers, Environment and Urban Systems, 2019. 75: p. 61-75.

61. Mei, L., et al., Dynamic risk assessment in healthcare based on Bayesian approach. Reliability Engineering \& System Safety, 2019.

62. Frazier, D.T., et al., Approximate Bayesian forecasting. International Journal of Forecasting, 2019. 35(2): p. 521-539.

63. Chan, T., et al., PCANet: A Simple Deep Learning Baseline for Image Classification? IEEE Transactions on Image Processing, 2015. 24(12): p. 5017-5032.

64. Song, G., et al., Music auto-tagging using deep Recurrent Neural Networks. Neurocomputing, 2018. 292: p. 104-110.

65. Mohamed, A., G.E. Dahl, and G. Hinton, Acoustic Modeling Using Deep Belief Networks. IEEE Transactions on Audio, Speech, and Language Processing, 2012. 20(1): p. 14-22.

66. Sedhain, S., et al. AutoRec: Autoencoders Meet Collaborative Filtering. in International Conference on World Wide Web. 2015.

67. Bai, Y., et al., Hourly PM2.5 concentration forecast using stacked autoencoder model with emphasis on seasonality. Journal of Cleaner Production, 2019. 224: p. 739-750.

68. Shang, J., et al., Inferring gas consumption and pollution emission of vehicles throughout a city. 2014: p. 1027-1036.

69. Zheng, Y., et al., Urban computing: concepts, methodologies, and applications. ACM Transactions on Intelligent Systems and Technology (TIST), 2014. 5(3): p. 38.

70. Qi, L., et al., Time-aware distributed service recommendation with privacy-preservation. Information Sciences, 2019. 480: p. 354-364.

71. Shuang, W. and H. Ya-Ru, Deep Learning Network for Multiuser Detection in Satellite Mobile Communication System. Computational Intelligence and Neuroscience, 2019. 2019. 